\title{
Effects of grape seed proanthocyanidin extract on renal injury in type 2 diabetic rats
}

\author{
LEI BAO, ZHAOFENG ZHANG, XIAOQIAN DAI, YE DING，YANFEI JIANG，YUJIE LI and YONG LI \\ Department of Nutrition and Food Hygiene, School of Public Health, Peking University, Beijing 100191, P.R. China
}

Received March 23, 2014; Accepted October 1, 2014

DOI: $10.3892 / \mathrm{mmr} .2014 .2768$

\begin{abstract}
Grape seed proanthocyanidin extract (GSPE) is known to be an effective natural polyphenol capable of removing free radicals in vivo. It has been reported that GSPE has biological functions including antioxidant, anti-cancer, anti-hyperglycemic, anti-radiation, and prevention and treatment of cardiovascular diseases. This study aims to investigate the effects of GSPE on renal injury in type 2 diabetic rats induced with low-dose streptozotocin and a high-carbohydrate/high-fat diet. Rats ( $n=12$ per group) were administered GSPE at either a low (125 mg/kg·bw), medium (250 mg/kg.bw) or high (500 mg/kg.bw) dose, while control rats and diabetes mellitus group rats received no specific treatment. After 16 weeks, GSPE slightly increased body weight and decreased food consumption, water intake and urine volume in rats. Diabetic rats treated with GSPE demonstrated decreased fasting blood glucose, serum insulin, HbA1c and systolic blood pressure $(\mathrm{P}<0.05)$. GSPE significantly improved renal function parameters, reduced the expression of tissue inhibitor of metalloproteinase- 1 and also increased the activity of matrix metalloproteinase-9. Moreover, GSPE (particularly at a dose of $500 \mathrm{mg} / \mathrm{kg} \cdot \mathrm{bw}$ ) increased the activity of antioxidant enzymes and reduced the levels of c-reactive proteins $(\mathrm{P}<0.01)$ in serum and the expression of tumor necrosis factor- $\alpha$, monocyte chemoattractant protein-1 and intercellular adhesion molecule-1 $(\mathrm{P}<0.05)$ in the kidney.
\end{abstract}

Correspondence to: Professor Yong Li, Department of Nutrition and Food Hygiene, School of Public Health, Peking University, 38 Xueyuan Road, Haidian, Beijing 100191, P.R. China

E-mail: liyongbmu@163.com

Abbreviations: FBG, fasting blood glucose; hs-CRP, high-sensitivity c-reactive protein; $\mathrm{SCr}$, serum creatinine; $\mathrm{CCr}$, endogenous creatinine clearance rate; BUN, blood urea nitrogen; SOD, superoxide dismutase; CAT, catalase; MDA, malondialdehyde; TNF- $\alpha$, tumor necrosis factor- $\alpha$; TIMP-1, tissue inhibitor of metalloproteinase-1; ICAM-1, intercellular adhesion molecule-1; MCP-1, monocyte chemoattractant protein-1; MMP-9, matrix metalloproteinase-9

Key words: diabetic nephropathy, antioxidative activities, rats, inflammatory, grape seed proanthocyanidin extract
These findings suggest that GSPE ameliorates renal injury in type 2 diabetic rats through its antioxidative activity and anti-inflammatory effects.

\section{Introduction}

The incidence of diabetes mellitus (DM) is increasing worldwide relentlessly and rapidly. Type 2 diabetes mellitus (T2DM) accounts for $90-95 \%$ of all cases. It was estimated that 92.4 million adults in China (20 years and older) suffered from diabetes in 2010 while a further 148 million people were in the pre-diabetic state (1). Diabetic nephropathy (DN) is the primary microvascular complication of DM, and is also a major cause of chronic renal failure. It is estimated that approximately $50 \%$ of DM patients will suffer complications with DN within ten years of diagnosis, and the proportion of cases of $\mathrm{DN}$ in end-stage renal failure (ESRF) has been increasing every year (2). In the United States, DN is the leading cause of ESRF (3). In addition, the speed of development to ESRF in patients with $\mathrm{DN}$ is approximately 14 times as fast as that of normal kidney diseases (4).

The pathogenesis of DN is complex and has not yet been fully elucidated. Hyperglycemia has been proven to be the major driving force of the progression of DN (5). A previous study has indicated that oxidative stress is one of the mechanisms by which hyperglycemia causes DN (6). Oxidative stress refers to the imbalance between the production of reactive oxygen species (ROS) and the ability of endogenous antioxidative systems to scavenge these ROS (7). It is a systemic process which may occur in any tissue. Under normal conditions, ROS produced in the cellular metabolism is efficiently scavenged by the intrinsic antioxidant defense mechanisms. However, in the hyperglycemic state of DM, excessive ROS are produced in renal tissue due to the damaged scavenging ability. Excessive ROS leads to renal histological changes and functional abnormalities of oxidizing lipids, proteins and nucleic acids. A previous study has revealed that oxidative stress plays a critical role in the development and progression of DN (8). Interventions could ameliorate DN through the reduction of oxidative stress and the increase of renal antioxidant enzyme activity (9).

Inflammation is a further significant risk factor of DN, and has been scrutinized in recent years. Currently, anti-inflammatory drugs are used in the management of DN. Studies have reported that kidney inflammation is crucial 
in promoting the development and progression of DN (10). Inflammation may be a key factor which is activated by the metabolic, biochemical and hemodynamic derangements known to exist in the diabetic kidney (11). Hyperglycemia, advanced glycoxidation end products (AGEs) and immune complexes in DM decrease the release of chemokines and the upregulation of cell adhesion molecules (12). These events promote the infiltration of renal monocytes and lymphocytes. One study on drugs which have significant effects on $\mathrm{DN}$ has reported that these drugs work through their anti-inflammatory effects (13). However, existing drugs only partially alleviate the inflammatory response, and do not deter the progress of DN. If efficacy and safety are taken into consideration, there are few anti-inflammation drugs that truly function in the treatment of DN patients. Therefore, it is of particular urgency to identify chemicals which are safe and effective to combat DN.

Grape seed proanthocyanidin extract (GSPE) is a potent antioxidant extracted from grape seeds and skins. GSPE is reported to be highly bioavailable and provides significantly greater protection against the damage of oxidative stress than vitamins $C$ and $E$ and $\beta$-carotene (14). GSPE may be rapidly absorbed by the gastrointestinal tract and reaches a peak level after $45 \mathrm{~min}$. It has a half-life of $5 \mathrm{~h}$. Fourteen percent of it is evacuated by the biliary system in $11 \mathrm{~h}$ while $70 \%$ is evacuated in the form of $\mathrm{CO}_{2}$, urine and feces after $24 \mathrm{~h}$. Another study demonstrated that GSPE mediated a protective effect against doxorubicin-induced cardiac injury through antioxidant, anti-inflammatory and antiapoptotic mechanisms (15). Therefore, the use of GSPE against oxidative stress and inflammation offers the possibility of a protective effect on renal injury induced by DM. Previous studies have demonstrated that GSPE protects against renal injury induced by T1DM (16). However, there is limited information on its effects on renal injury induced by T2DM. This study aims to identify its effects and possible mechanisms.

\section{Materials and methods}

Materials and reagents. GSPE (lot no. 1003007-24) was donated by JF-Natural Limited Company (Tianjin, China). The proanthocyanidin content was $95 \%$ when analyzed using high-performance liquid chromatography with gas chromatography-mass spectrometry detection. It contained $56 \%$ dimers, $12 \%$ trimers, $6.6 \%$ tetramer and small amounts of other polymeric forms. The high-carbohydrate/high-fat diet was purchased from Beijing Ke'ao Limited Company (Beijing, China), and included $66 \%$ basal diet, $15 \%$ lard, $10 \%$ sucrose, $6 \%$ casein and $3 \%$ egg yolk.

Commercial kits used for the determination of superoxide dismutase (SOD), catalase (CAT), malondialdehyde (MDA) and protein in kidney were purchased from Jiancheng Institute of Biotechnology (Nanjing, China). The antibodies of tumor necrosis factor- $\alpha$ (TNF- $\alpha)$, monocyte chemoattractant protein-1 (MCP-1), intercellular adhesion molecule-1 (ICAM-1) and tissue inhibitor of metalloproteinase-1 (TIMP-1) were purchased from Zhongshanjinqiao Biotechnology Limited Company (Beijing, China). Matrix metalloproteinase-9 (MMP-9) was purchased from Santa Cruz Biotechnology, Inc. (Santa Cruz, CA, USA). Ethanol, acetaldehyde and isopropanol were of analytical grade and purchased from Beijing Chemical Company (Beijing, China).

Animals and treatment. Seventy male Sprague-Dawley rats (6 weeks old, weighing 200-250 g) were obtained from the Animal Service of the Health Science Center, Peking University, China. The rats were kept in a filter-protected, air-conditioned room with controlled temperature $\left(21-25^{\circ} \mathrm{C}\right)$, relative air humidity (40-50\%) and a 12-h light/12-h dark cycle (lights on between 07.30 and 19.30). Animal treatment and maintenance were carried out strictly in accordance with the Principles of Laboratory Animal Care (NIH publication no. 85-23, 1985) and the guidelines of the Peking University Animal Research Committee.

Experimental design. Rats were randomly divided into two groups. Group $1(\mathrm{n}=12$, normal control) was fed the basal diet. Group $2(n=58)$ was fed a high-carbohydrate/high-fat diet for 4 weeks. Then after fasting for $15 \mathrm{~h}$, rats in group 2 were intraperitoneally injected with streptozotocin (STZ; $0.01 \mathrm{~mol} / 1,30 \mathrm{mg} / \mathrm{kg} \cdot \mathrm{bw})$. Another STZ injection was administered after 7 days. STZ was freshly dissolved in ice-cold citrate buffer ( $\mathrm{pH} 4.5)$. Rats in group 1 were injected with the citrate buffer vehicle at the same time. Fourteen days after the STZ injection, the fasting serum glucose of rats in group 2 was tested. Diabetes was defined as fasting serum glucose $>11.1 \mathrm{mmol} / 1$ in caudal vein blood and rats having the symptoms of polyuria, polyphagia and emaciation. A total of 48 rats in group 2 satisfied the above conditions and were used in the next experiments. The diabetic rats $(n=48)$ were randomly divided into four groups ( $\mathrm{n}=12$ in each): one DM control group and three groups for low $(125 \mathrm{mg} / \mathrm{kg} \cdot \mathrm{bw})$, medium $(250 \mathrm{mg} / \mathrm{kg} \cdot \mathrm{bw})$ and high $(500 \mathrm{mg} / \mathrm{kg} \cdot \mathrm{bw})$ doses of GSPE. Distilled water was used as the solvent for GSPE. The rats in the GSPE intervention groups were given GSPE intragastrically while the others received an equal volume of distilled water during the experimental period. The study lasted for a total of 16 weeks. Food consumption, water intake and urine volume of rats were measured through metabolic cages. Systolic blood pressures of all rats were measured. Blood was collected from the femoral artery and the rats were then sacrificed by cervical dislocation after collecting $24 \mathrm{~h}$ volume urine. Serum was separated by centrifugation at 3,000 rpm for $15 \mathrm{~min}$. Portions of the kidney were then obtained for biochemical assays.

Biochemical assays. Systolic blood pressure was measured by the BP-98A intelligent non-invasive blood pressure monitor (Softron Biotechnology Limited Company, Beijing, China). The levels of fasting blood glucose (FBG) and high-sensitivity c-reactive protein (hs-CRP) in serum were detected using an automatic biochemistry analyzer (Hitachi, Tokyo, Japan), as were serum creatinine ( $\mathrm{SCr}$ ) and blood urea nitrogen (BUN) in urine. SOD and CAT activity and the MDA level in renal tissues were determined by detection kits according to the manufacturer's instructions.

Western blot analysis. Total protein was extracted from the frozen renal tissues using RIPA lysis buffer (1\% Triton X-100, $1 \%$ deoxycholate, $0.1 \%$ SDS) and $1 \mathrm{mM}$ phenylmethanesulfonyl 
Table I. Effect of grape seed proanthocyanidin extract on body weight, food intake, water intake and urine volume in rats after 16 weeks.

\begin{tabular}{lccccc}
\hline Parameters & Normal rats & DM rats & Low GSPE rats & Medium GSPE rats & High GSPE rats \\
\hline Initial body weight $(\mathrm{g})$ & $425.70 \pm 5.87$ & $442.23 \pm 10.15^{\mathrm{a}}$ & $452.43 \pm 12.78$ & $440.22 \pm 8.17$ & $454.50 \pm 9.42$ \\
Final body weight $(\mathrm{g})$ & $585.38 \pm 13.23$ & $415.78 \pm 9.45^{\mathrm{a}}$ & $434.53 \pm 10.89$ & $447.65 \pm 12.74^{\mathrm{c}}$ & $450.63 \pm 11.67^{\mathrm{c}}$ \\
Initial food intake $(\mathrm{g} / \mathrm{d})$ & $16.86 \pm 0.88$ & $23.43 \pm 1.24^{\mathrm{a}}$ & $19.16 \pm 1.56$ & $21.13 \pm 0.98$ & $20.16 \pm 1.42$ \\
Final food intake $(\mathrm{g} / \mathrm{d})$ & $15.50 \pm 0.56$ & $24.67 \pm 1.55^{\mathrm{b}}$ & $27.50 \pm 1.01$ & $20.75 \pm 1.32^{\mathrm{c}}$ & $18.14 \pm 1.42^{\mathrm{c}}$ \\
Initial water intake $(\mathrm{ml} / \mathrm{d})$ & $40.21 \pm 4.32$ & $80.76 \pm 5.05^{\mathrm{a}}$ & $78.14 \pm 9.97$ & $82.14 \pm 7.74$ & $86.13 \pm 6.94$ \\
Final water intake $(\mathrm{ml} / \mathrm{d})$ & $35.77 \pm 5.11$ & $121.67 \pm 11.67^{\mathrm{b}}$ & $115.71 \pm 8.65$ & $78.59 \pm 5.67^{\mathrm{d}}$ & $71.57 \pm 5.43^{\mathrm{d}}$ \\
Initial urine volume $(\mathrm{ml} / \mathrm{d})$ & $7.57 \pm 1.73$ & $36.50 \pm 8.39^{\mathrm{b}}$ & $33.26 \pm 6.38$ & $40.58 \pm 2.32$ & $38.85 \pm 3.59$ \\
Final urine volume $(\mathrm{ml} / \mathrm{d})$ & $6.71 \pm 1.95$ & $100.80 \pm 9.96^{\mathrm{b}}$ & $90.13 \pm 10.55$ & $70.83 \pm 10.50^{\mathrm{c}}$ & $55.75 \pm 5.12^{\mathrm{d}}$ \\
\hline
\end{tabular}

DM, diabetes mellitus; GSPE, grape seed proanthocyanidin extract. Data are expressed as the mean \pm standard deviation of 12 rats per group. ${ }^{\mathrm{a}} \mathrm{P}<0.05,{ }^{\mathrm{b}} \mathrm{P}<0.01$ versus normal control rats; ${ }^{\mathrm{c}} \mathrm{P}<0.05$, ${ }^{\mathrm{d}} \mathrm{P}<0.01$ versus $\mathrm{DM}$ control rats.

fluoride. Following ultrasonication for $5 \mathrm{~min}$, extracts were centrifuged at $12,000 \mathrm{rpm}$ for $15 \mathrm{~min}$ at $4^{\circ} \mathrm{C}$, and the supernatants containing protein were retained. The concentrations of protein were measured with the bicinchoninic acid method (Beyotime $^{\circledR}$, Institute of Biochemistry, China). Then $40-\mu \mathrm{g}$ protein samples were resolved on $15 \%$ Tris-glycine polyacrylamide minigels for TNF- $\alpha$, MCP-1 and TIMP-1 and 5\% for ICAM-1 and MMP-9, and then transferred to polyvinylidene fluoride (PVDF) membranes. Membranes were blocked with $5 \%$ skimmed milk in TBST for $1 \mathrm{~h}$. Primary antibodies were used overnight at $4^{\circ} \mathrm{C}$ and dilutions were as follows: TNF- $\alpha$ (1:200), MCP-1 (1:200), TIMP-1 (1:200), MMP-9 (1:200) and ICAM-1 (1:500), all from Santa Cruz. Secondary antibodies were used at the following dilutions: goat antibody to mouse $\operatorname{IgG}(1: 5000)$ and goat anti-rabbit (1:5000), both from ZSGB-BIO, Beijing, China. Signals were visualized using the enhanced chemiluminescence system and analyzed densitometrically using Image Pro Plus version 6.0 software (Media Cybernetics, Inc., Silver Spring, MD, USA). PVDF membranes were reprobed with $\beta$-actin (at 1:1000, Cell Signaling Technology, Inc., Beverly, MA, USA) to verify equal protein loading on the gel.

Statistical analysis. The data are expressed as the mean \pm standard deviations. The analyses were carried out with SPSS 13.0 statistical software (SPSS, Inc., Chicago, IL, USA). Data were first tested for homogeneity and then evaluated by means of a one-way ANOVA test followed by Tukey's post hoc least significant difference test, if variances were equal, or Tamhane's T3 test, if variances were not equal. $\mathrm{P}<0.05$ was considered to indicate a statistically significant result.

\section{Results}

Body weight, food consumption, water intake and urine volume values. As shown in Table I, the body weight was lower in DM rats than in normal rats at the end of the study $(\mathrm{P}<0.05)$. Food consumption, water intake and urine volume were increased in DM rats compared with normal rats throughout the study period ( $\mathrm{P}<0.01$ for each). However, GSPE treatment slightly increased body weight and decreased food consumption, water intake and urine volume in a dose-dependent manner.

Fasting blood glucose, serum insulin, HbAlc and systolic blood pressure values. As shown in Fig. 1, FBG, serum insulin, $\mathrm{HbAlc}$ and systolic blood pressure were markedly increased in $\mathrm{DM}$ control rats compared with normal control rats $(\mathrm{P}<0.05)$. Following administration of GSPE (particularly at a dose of $500 \mathrm{mg} / \mathrm{kg} \cdot \mathrm{bw}$ ), the above parameters were decreased to a certain extent.

Renal function index values. BUN, SCr, creatinine clearance $(\mathrm{CCr}), 24-\mathrm{h}$ urine protein and kidney index (kidney weight/body weight) were used to evaluate renal injury. From Table II it may be noted that some renal injury occurred in DM control rats including renal hypertrophy and renal dysfunction $(\mathrm{P}<0.01$ or $\mathrm{P}<0.05$ compared with normal control rats). However, GSPE treatment was able to improve these renal function parameters $(\mathrm{P}<0.01$ or $\mathrm{P}<0.05)$.

MMP-9 and TIMP-1 expression in renal tissues. As shown in Fig. 2, low-dose streptozotocin and a high-carbohydrate/high-fat diet treatment significantly decreased the expression of MMP-9 and increased the expression of TIMP-1 compared with that observed in the normal control rats $(\mathrm{P}<0.05)$. The $500 \mathrm{mg} / \mathrm{kg} \cdot \mathrm{bw}$ GSPE treatment markedly increased the expression of MMP-9 and reduced the expression of TIMP-1 when compared with diabetic control rats $(\mathrm{P}<0.05)$.

SOD and CAT activity and MDA levels in renal tissues. Parameters of oxidative stress are shown in Table III. The activity of SOD and CAT in the kidneys of the DM control rats was significantly lower than that in normal control rats $(\mathrm{P}<0.01)$ while MDA levels were significantly higher $(\mathrm{P}<0.05)$. The SOD and CAT activity in high-dose GSPE rats was significantly higher than that in $\mathrm{DM}$ control rats $(\mathrm{P}<0.01$ and $\mathrm{P}<0.05$, respectively). The MDA levels in high-dose GSPE-treated 
Table II. Effect of grape seed proanthocyanidin extract on 24-h urine protein, blood urea nitrogen, serum creatinine, creatinine clearance and renal mass in rats.

\begin{tabular}{lrcrrr}
\hline Parameters & Normal rats & DM rats & Low GSPE rats & Medium GSPE rats & High GSPE rats \\
\hline 24-h urine protein (mg) & $77.96 \pm 3.83$ & $524.76 \pm 8.75^{\mathrm{b}}$ & $539.50 \pm 6.30$ & $445.39 \pm 4.59^{\mathrm{c}}$ & $231.04 \pm 4.07^{\mathrm{d}}$ \\
BUN (mmol/l) & $4.38 \pm 0.26$ & $12.25 \pm 5.09^{\mathrm{b}}$ & $7.97 \pm 0.89$ & $7.15 \pm 0.77$ & $5.12 \pm 1.56^{\mathrm{d}}$ \\
SCr $(\mu \mathrm{mol} / \mathrm{l})$ & $30.38 \pm 8.39$ & $48.47 \pm 10.32^{\mathrm{a}}$ & $43.12 \pm 9.66$ & $39.63 \pm 11.21$ & $31.12 \pm 9.95^{\mathrm{d}}$ \\
CCr $(\mathrm{mmol} / \mathrm{l})$ & $2.03 \pm 0.23$ & $6.79 \pm 1.04^{\mathrm{b}}$ & $6.03 \pm 0.53$ & $5.07 \pm 0.60$ & $3.35 \pm 1.01^{\mathrm{d}}$ \\
Kidney weight/body weight (\%) & $0.46 \pm 0.02$ & $0.76 \pm 0.02^{\mathrm{b}}$ & $0.82 \pm 0.02$ & $0.74 \pm 0.04$ & $0.54 \pm 0.02^{\mathrm{d}}$ \\
\hline
\end{tabular}

DM, diabetes mellitus; GSPE, grape seed proanthocyanidin extract; BUN, blood urea nitrogen; SCr, serum creatinine; $\mathrm{CCr}$, creatinine clearance. Data are expressed as the mean \pm standard deviation of 12 rats per group. ${ }^{\mathrm{a}} \mathrm{P}<0.05,{ }^{\mathrm{b}} \mathrm{P}<0.01$ versus normal control rats; ${ }^{\mathrm{c}} \mathrm{P}<0.05$, ${ }^{\mathrm{d}} \mathrm{P}<0.01$ versus DM control rats.
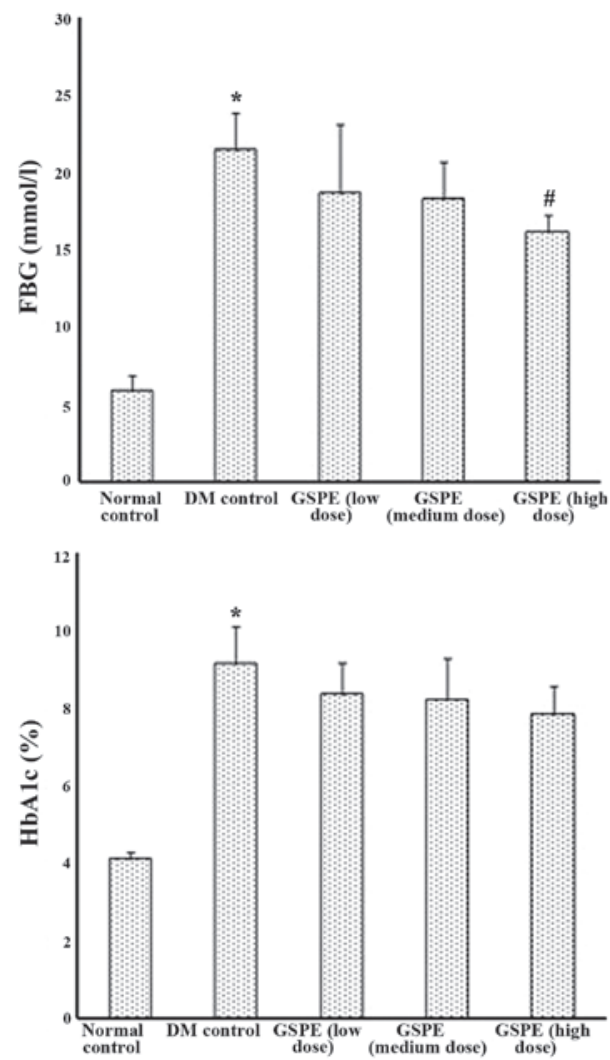
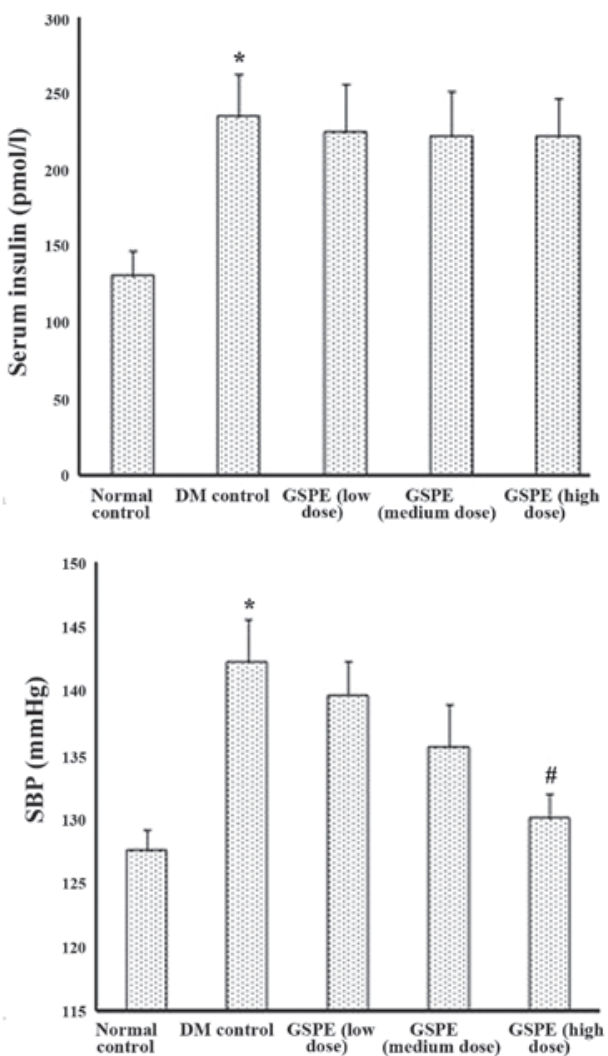

Figure 1. Effects of grape seed proanthocyanidin extract (GSPE) on fasting blood glucose (FBG), serum insulin, HbAlc and systolic blood pressure (SBP) in rats. DM, diabetes mellitus. Data are expressed as the mean \pm standard deviation of 12 rats per group. ${ }^{*} \mathrm{P}<0.05$ versus normal control rats; ${ }^{*} \mathrm{P}<0.05$ versus $\mathrm{DM}$ control rats.

rats were significantly lower than those in DM control rats $(\mathrm{P}<0.01)$.

Serum high sensitivity c-reactive protein levels and expression of TNF- $\alpha, M C P-1$ and ICAM-1 in renal tissues. Parameters of inflammation were determined as shown in Figs. 3 and 4. Compared with normal control rats, serum hs-CRP in DM control rats was significantly increased $(\mathrm{P}<0.01)$, while in GSPE medium- and high-dose groups it was significantly lower than in $\mathrm{DM}$ control rats $(\mathrm{P}<0.01)$. Inflammatory cytokines, including TNF- $\alpha$, MCP-1 and ICAM-1, were all significantly elevated in the kidneys of DM control rats when compared with normal controls ( $\mathrm{P}<0.05$ for each). However, GSPE (particularly at a dose of $500 \mathrm{mg} / \mathrm{kg} \cdot \mathrm{bw}$ ) decreased their expression when compared with the DM control group ( $\mathrm{P}<0.05$ for each).

\section{Discussion}

Due to its poor prognosis and the rapidly increasing number of cases, type $2 \mathrm{DN}$ has raised great concern. In this study, we successfully built a T2DM model of rats using a high-carbohydrate/high-fat diet for 4 weeks and two low-dose streptozotocin injections. Following 16 weeks of treatment, 
Table III. Effect of grape seed proanthocyanidin extract on superoxide dismutase and catalase activity and malondialdehyde levels in renal tissues of rats.

\begin{tabular}{lrcccr}
\hline Parameters & Normal rats & DM rats & Low GSPE rats & Medium GSPE rats & High GSPE rats \\
\hline SOD (U/mg·pro) & $69.45 \pm 5.27$ & $53.71 \pm 3.45^{\mathrm{b}}$ & $60.72 \pm 10.10$ & $62.58 \pm 4.35$ & $67.78 \pm 7.11^{\mathrm{d}}$ \\
CAT (U/mg·pro) & $38.46 \pm 2.75$ & $28.01 \pm 2.64^{\mathrm{b}}$ & $30.70 \pm 2.76$ & $32.38 \pm 5.14$ & $35.65 \pm 2.32^{\mathrm{c}}$ \\
MDA (nmol/mg·pro) & $0.59 \pm 0.18$ & $1.15 \pm 0.31^{\mathrm{a}}$ & $0.82 \pm 0.16$ & $0.72 \pm 0.16$ & $0.53 \pm 0.25^{\mathrm{d}}$ \\
\hline
\end{tabular}

DM, diabetes mellitus; GSPE, grape seed proanthocyanidin extract; SOD, superoxide dismutase, CAT, catalase; MDA, malondialdehyde. Data are expressed as the mean \pm standard deviation of 12 rats per group. ${ }^{a} \mathrm{P}<0.05,{ }^{\mathrm{b}} \mathrm{P}<0.01$ versus normal control rats; ${ }^{\mathrm{C}} \mathrm{P}<0.05,{ }^{\mathrm{d}} \mathrm{P}<0.01 \mathrm{versus} \mathrm{DM}$ control rats.
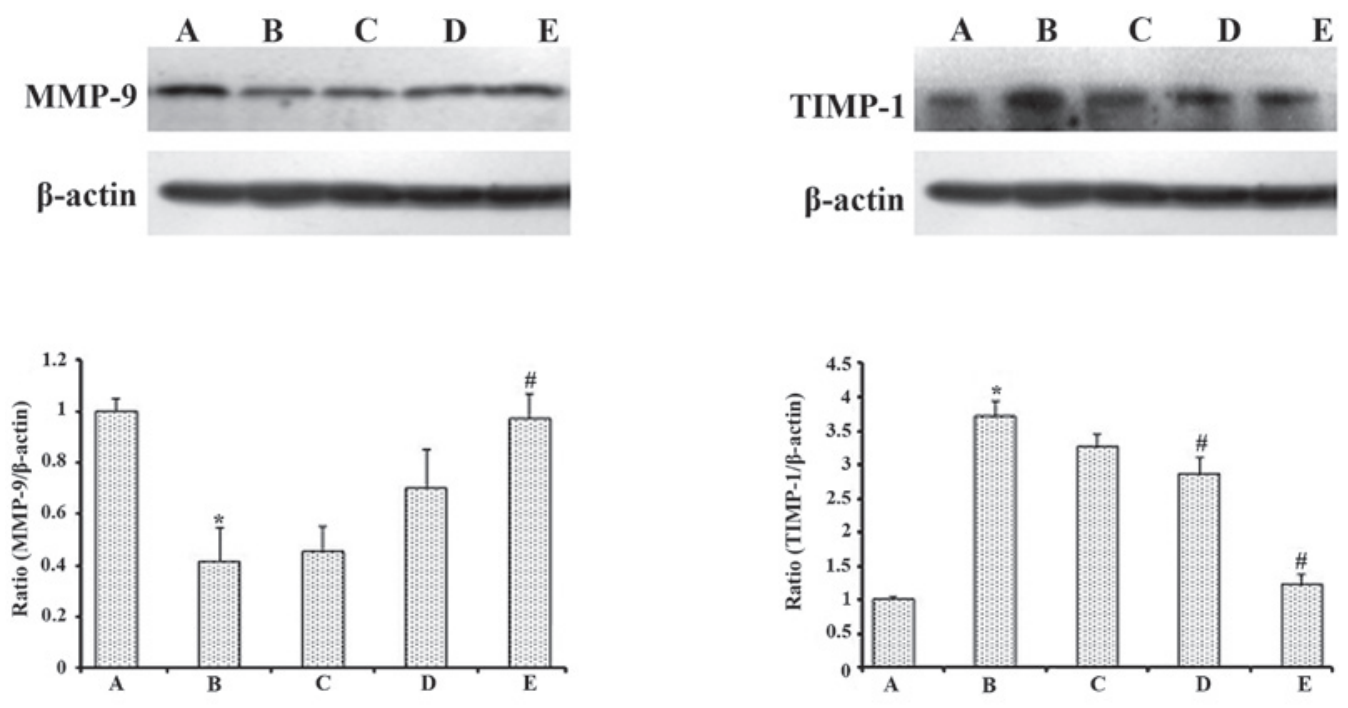

Figure 2. Effect of grape seed proanthocyanidin extract (GSPE) on matrix metalloproteinase-9 (MMP-9) and tissue inhibitor of metalloproteinase-1 (TIMP-1) in renal tissues of rats. $\beta$-actin protein levels were used as a control. Data are expressed as the mean \pm standard deviation of four rats per group. "P $<0.05$ versus normal control rats; ${ }^{\#} \mathrm{P}<0.05$ versus diabetes mellitus (DM) control rats. (A) Normal control group; (B) DM control group; (C) low-dose GSPE group; (D) medium-dose GSPE group; (E) high-dose GSPE group.

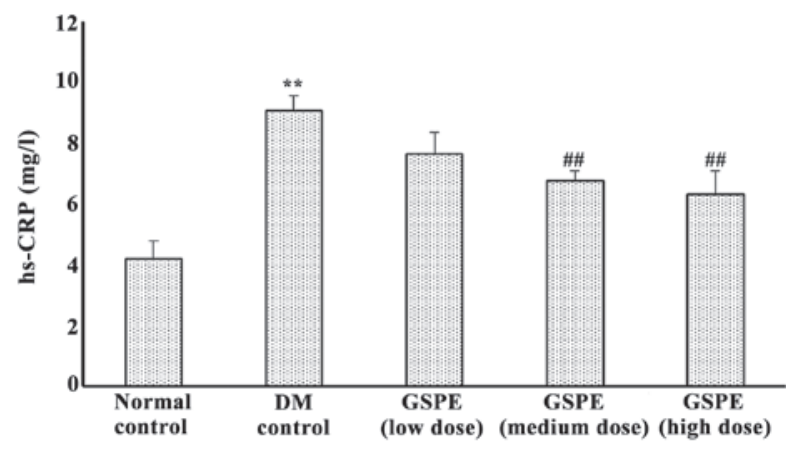

Figure 3. Effect of grape seed proanthocyanidin extract (GSPE) on serum high-sensitivity c-reactive protein (hs-CRP) levels in rats. Data are expressed as the mean \pm standard deviation of 12 rats per group. ${ }^{* *} \mathrm{P}<0.01$ versus normal control rats; ${ }^{\#} \mathrm{P}<0.01$ versus diabetes mellitus (DM) control rats.

GSPE was demonstrated to improve renal function, decrease the levels of FBG, serum insulin, HbA1c and systolic blood pressure, and suppress oxidative stress and inflammatory response.

At the end of this study, nephritic structural and functional damages were observed in diabetic control rats as demonstrated by the BUN, $\mathrm{SCr}$, endogenous $\mathrm{CCr}$, 24-h proteinuria, kidney weight/body weight, and expression of MMP-9 and TIMP-1. Increased glomerular filtration rate and proteinuria are the main physical signs of DN. BUN, SCr, CCr and 24-h urine protein are the main indicators of renal function. In the present study, the BUN, $\mathrm{SCr}, \mathrm{CCr}$ and 24-h urine protein of $\mathrm{DM}$ rats were significantly higher than those in normal rats, which implied glomerular hyperfiltration and renal dysfunction in the DM rats. However, GSPE improved these renal function parameters. This is similar to the results observed in a previous study by Li et al (17). These authors also indicated that GSPE was capable of decreasing proteinuria, attenuating the progression of nephropathy in diabetic rats.

Glomerular extracellular matrix (ECM) accumulation and base-membrane thickness are the main pathological changes in DN (18). MMP-9 is considered to be essential for ECM turnover in kidneys, and the downregulation of MMP-9 levels has been demonstrated in streptozotocin-induced diabetic rats $(19,20)$. Previous studies have indicated that an imbalance between MMPs and their inhibitors (TIMPs) contributes to nephropathy and that they may be potential targets for therapeutic interventions $(21,22)$. In the present study, decreased MMP-9 activity and increased TIMP-1 activity were observed in diabetic rats. GSPE promoted the upregulation of MMP-9 

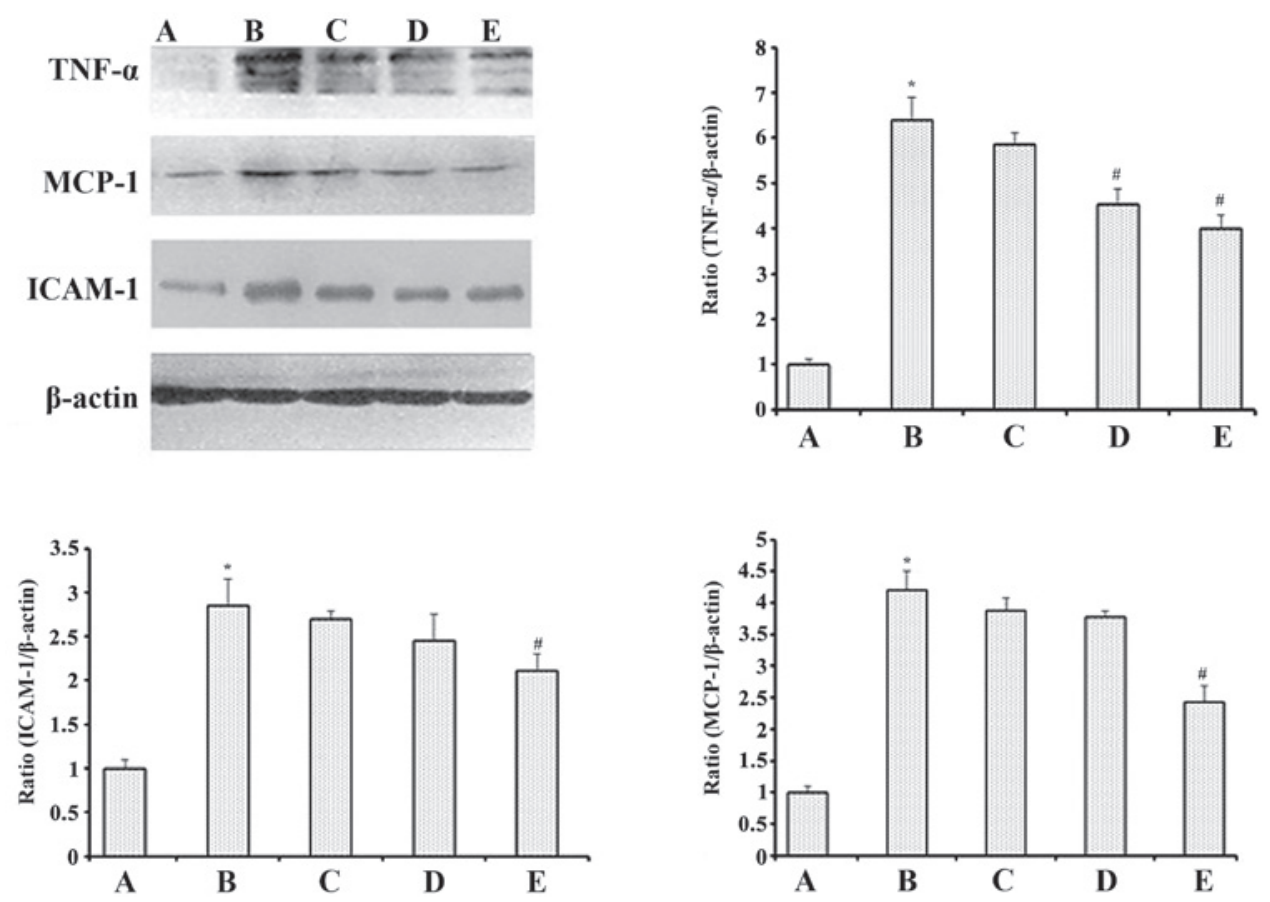

Figure 4. Effect of grape seed proanthocyanidin extract (GSPE) on tumor necrosis factor- $\alpha$ (TNF- $\alpha$ ), monocyte chemoattractant protein-1 (MCP-1) and intercellular adhesion molecule-1 (ICAM-1) in renal tissues of rats. $\beta$-actin protein levels were used as a control. Data are expressed as the mean \pm standard deviation of four rats per group. ${ }^{~} \mathrm{P}<0.05$ versus normal control rats; ${ }^{*} \mathrm{P}<0.05$ versus diabetes mellitus (DM) control rats. (A) Normal control group; (B) DM control group; (C) low-dose GSPE group; (D) medium-dose GSPE group; (E) high-dose GSPE group.

and downregulation of TIMP-1, which may be a potential mechanism of the protective effect. This finding may be significant as there are few reports on MMPs and their inhibitors in studies involving GSPE in DN.

Hyperglycemia is a typical feature of DM, which is also one of the most common symptoms. Long-term hyperglycemia is the main cause of DN, thereby imposing a great risk to patients. One prospective diabetes study from the UK demonstrated that the risk of microalbuminuria and clinical DN in DM patients could be significantly reduced if intensive treatment was administered to control the levels of blood glucose (23). Pinent et al (24) observed that GSPE had a hypoglycemic effect on STZ-induced diabetic rats by delaying the absorption of glucose in intestines. In addition, El-Alfy et al (25) demonstrated that GSPE could lower hyperglycemia in mice induced by alloxan monohydrate in an antagonistic manner. GSPE, as an effective antioxidant, protects islet tissue from damage, and may also promote residual islet B cells to secrete insulin. A previous study revealed that $250 \mathrm{mg} / \mathrm{kg} \cdot \mathrm{bw}$ GSPE could not decrease FBG and HbAlc levels in diabetic rats (26). In the present study, we noted that $500 \mathrm{mg} / \mathrm{kg} \cdot \mathrm{bw}$ GSPE slightly decreased the levels of FBG.

Hypertension is always accompanied by hyperglycemia, and is another major cause of DN. Glomerular capillary pressure due to high blood pressure may cause increased capillary tension and thickening of the arterial wall, thus leading to ischemia and endothelial cell damage. Then mesangial cells and matrix proliferate and promote glomerular metabolism and compensatory hypertrophy, which eventually results in glomerulosclerosis. This damage could cause the blood pressure to increase in turn, creating a vicious cycle thereafter. Therefore, controlling the blood pressure to a normal level is also essential in the treatment of DN. In our study, the systolic blood pressure in GSPE-treated rats was significantly lower than that in normal rats, which indicated that GSPE was able to reduce blood pressure. Cui et al (27) fed ouabain-induced hypertensive rats with GSPE and noted a lowered blood pressure, making GSPE a potential natural reagent in hypertension treatment. Li et al (17) also observed a similar result. GSPE may enhance the production and release of nitric oxide, reduce the expression of endothelin-1 in the vascular endothelium, and thus have an effect on hypertension (28).

Increased oxidative stress in DN induces a number of ROS, including peroxide hydrogen $\left(\mathrm{H}_{2} \mathrm{O}_{2}\right)$, superoxide anion $\left(\mathrm{O}^{2-}\right)$ and hydroxyl radical $(\cdot \mathrm{OH})$. ROS activate nuclear transcription factor- $\kappa \mathrm{B}$ and activator protein-1 by activating protein kinase $\mathrm{C}(\mathrm{PKC})$ and mitogen-activated protein, which results in large amounts of cytokines and growth factors in the kidney (29,30). Microvascular endothelial cells are damaged as a result, and a number of negative consequences induced by oxidative stress, such as mitochondrial DNA deletions, will accelerate the development of DN $(31,32)$. Numerous studies have suggested that antioxidant therapy reduces renal injury in DN (33-35). SOD is the main antioxidant enzyme in vivo, while CAT is an enzyme scavenger decomposing hydrogen peroxide into hydrogen and water, and MDA is the most abundant by-product of lipid peroxidation. These three may be considered as indicators of oxidative stress. The present results reveal that DM rats possessed a higher MDA level and lower SOD and CAT activity levels in the kidney than those of normal control rats, which confirmed the involvement of oxidative stress in renal injury induced by DM. It was also noted that GSPE increased the activity of SOD and CAT, but decreased the levels of MDA. In other 
words, GSPE reduced the levels of ROS and protected the kidneys from injury induced by oxidative stress, which may be one of the potential mechanisms by which it exerts its beneficial effects on DN.

Possibly the most significant results in this study were the notable anti-inflammatory effects of GSPE and its pivotal role in the treatment of DN. An increasing number of inflammatory signal pathways and cytokines are being investigated and deemed new molecular targets for treating DN. In the progress of DN, certain pro-inflammatory cytokines and ROS become activated, which induces mesangial cells to secrete type IV collagen, laminin and fibronectin, and leads to glomerulosclerosis $(36,37)$. Moreover, inflammation is involved in the activation of certain signal transduction pathways in vivo, including the polyol, AGE, ROS and PKC pathways, which promotes the expression of the specific cytokines and induces the expression of the gene within the cell as well as protein dysfunction, eventually leading to the occurrence of microvascular complications (38). The present study revealed a severe inflammatory reaction in the serum and kidney. The levels of TNF- $\alpha$, MCP-1 and ICAM-1 were all significantly higher than those of normal controls. Previous studies have reported that renal injury was attenuated when their levels were decreased (39-41). One of mechanisms of protecting the kidney may be that GSPE decreased the levels of inflammatory cytokines.

In addition, inflammation and oxidative stress are not assumed to take effect separately. They are interrelated and interactive with each other in a number of diseases, including hypertension and cardiovascular disease $(42,43)$. However, information on the correlation between oxidative stress and inflammation in DN is limited. Further studies are necessary to identify whether either of these play a significant role in the treatment of DN.

In summary, this study indicated that oxidative stress and inflammatory reaction may, at least in part, cause renal injury in T2DM rats. GSPE may alleviate renal injury and exert its antioxidative effect in vivo by increasing the activity of SOD and CAT, decreasing the levels of MDA, and decreasing the levels of inflammatory cytokines including hs-CRP, TNF- $\alpha$, MCP-1 and ICAM-1. However, there are several inflammatory pathways in vitro. Further studies are required to investigate the exact inflammatory pathways by which GSPE protects against renal injury, as well as its specific mechanism of action.

\section{Acknowledgements}

This study was supported by research grants from the National Natural Science Foundation of China (81102116 and 81072293).

\section{References}

1. Yang W, Lu J, Weng J, et al: Prevalence of diabetes among men and women in China. New Engl J Med 362: 1090-1101, 2010.

2. Ceriello A: New insights on oxidative stress and diabetic complications may lead to a "causal" antioxidant therapy. Diabetes Care 26: 1589-1596, 2003.

3. Collins AJ, Kasiske B, Herzog C, et al: Excerpts from the United States Renal Data System 2003 Annual Data Report: atlas of end-stage renal disease in the United States. Am J Kidney Dis 42: A5-A7, S1-S230, 2003.

4. Caramori ML, Fioretto P and Mauer M: Low glomerular filtration rate in normoal buminuric type 1 diabetic patients: an indicator of more advanced glomerular lesions. Diabetes 52: 1036-1040, 2003.
5. Wada $\mathbf{J}$ and Makino $\mathrm{H}$ : Inflammation and the pathogenesis of diabetic nephropathy. Clin Sci (Lond) 124: 139-152, 2013.

6. Ha H, Yu MR and Kim KH: Melatonin and taurine reduce early glomerulopathy in diabetic rats. Free Radic Biol Med 26: 944-950, 1999.

7. Li SY, Fu ZJ and Lo AC: Hypoxia-induced oxidative stress in ischemic retinopathy. Oxid Med Cell Longev 2012: e426769, 2012.

8. Pan HZ, Zhang L, Guo MY, et al: The oxidative stress status in diabetes mellitus and diabetic nephropathy. Acta Diabetol 47: 71-76, 2010

9. Mansouri E, Panahi M, Ghaffari MA, et al: Effects of grape seed proanthocyanidin extract on oxidative stress induced by diabetes in rat kidney. Iran Biomed J 15: 100-106, 2011.

10. Kanasaki K, Taduri G and Koya D: Diabetic nephropathy: the role of inflammation in fibroblast activation and kidney fibrosis. Front Endocrinol (Lausanne) 4: 7, 2013.

11. Lim AK and Tesch GH: Inflammation in diabetic nephropathy. Mediators Inflamm 2012: 146-154, 2012.

12. Rivero A, Mora C, Muros M, et al: Pathogenic perspectives for the role of inflammation in diabetic nephropathy. Clin Sci (Lond) 116: 479-492, 2009.

13. Gui D, Huang J, Guo Y, et al: Astragaloside IV ameliorates renal injury in streptozotocin-induced diabetic rats through inhibiting NF-kB-mediated inflammatory genes expression. Cytokine 61: 970-977, 2013

14. Zhang XY, Li WG, Wu YJ, et al: Proanthocyanidin from grape seeds enhances doxorubicin-induced antitumor effect and reverses drug resistance in doxorubicin-resistant K562/DOX cells. Can J Physiol Pharm 83: 309-318, 2005.

15. Boghdady NA: Antioxidant and antiapoptotic effects of proanthocyanidin and ginkgo biloba extract against doxorubicin-induced cardiac injury in rats. Cell Biochem Funct 31: 344-351, 2013.

16. Mansouri E, Panahi M, Ghaffari MA and Ghorbani A: Effects of grape seed proanthocyanidin extract on oxidative stress induced by diabetes in rat kidney. Iran Biomed J 15: 100-106, 2011.

17. Li X, Xiao Y, Gao H, et al: Grape seed proanthocyanidins ameliorate diabetic nephropathy via modulation of levels of AGE, RAGE and CTGF. Nephron Exp Nephrol 111: e31-e41, 2009.

18. Kolset SO, Reinholt FP and Jenssen T: Diabetic nephropathy and extracellular matrix. J Histochem Cytochem 60: 976-986, 2012.

19. Furness PN: Basement membrane synthesis and degradation. J Pathol 183: 1-3, 1997.

20. Wu K, Setty S, Mauer SM, et al: Altered kidney matrix gene expression in early stages of experimental diabetes. Acta Anat (Basel) 158: 155-165, 1997.

21. Han SY, Jee YH, Han KH, et al: An imbalance between matrix metalloproteinase-2 and tissue inhibitor of matrix metalloproteinase-2 contributes to the development of early diabetic nephropathy. Nephrol Dial Transplant 21: 2406-2416, 2006.

22. Hadler-Olsen E, Winberg JO, Reinholt FP, et al: Proteases in Plasma and Kidney of db/db Mice as Markers of Diabetes-Induced Nephropathy. ISRN Endocrinol 2011: e832642, 2011.

23. Efficacy of atenolol and captopril in reducing risk of macrovascular and microvascular complications in type 2 diabetes: UKPDS 39. UK Prospective Diabetes Study Group. BMJ 317: 713-720, 1998

24. Pinent M, Blay M, Bladé MC, et al: Grape seed-derived procyanidins have an antihyperglycemic effect in streptozotocin-induced diabetic rats and insulin-omimetic activity in insulin-sensitive cell lines. Endocrinology 145: 4985-4990, 2004.

25. El-Alfy AT, Ahmed AA and Fatani AJ: Protective effect of red grape seeds proanthocyanidins against induction of diabetes by alloxan in rats. Pharmacol Res 52: 264-270, 2005.

26. Li BY, Cheng M, Gao HQ, et al: Back-regulation of six oxidative stress proteins with grape seed proanthocyanidin extracts in rat diabetic nephropathy. J Cell Biochem 104: 668-679, 2008.

27. Xiaopei Cui, Xiangju Liu, Hua Feng, et al: Grape seed proanthocyanidin extracts enhance endothelial nitric oxide synthase expression through 5'-AMP activated protein kinase/Surtuin 1-Krüpple like factor 2 pathway and modulate blood pressure in ouabain induced hypertensive rats. Biol Pharm Bull 35: 2192-2197, 2012.

28. Wu S, Guo R, Guo R, et al: Effects of grape seed proanthocyanidin extracts on renal vascular hypertension in rats. Chin J Pathophysiol 27: 593-595, 2011.

29. Ha $\mathrm{H}$ and Lee HB: Reactive oxygen species as glucose signaling molecules in mesangial cells cultured under high glucose. Kidney Int Suppl 77: S19-S25, 2000. 
30. Scivittaro V, Ganz MB and Weiss MF: AGEs induce oxidative stress and activate protein kinase C-beta (II) in neonatal mesangial cells. Am J Physiol Renal Physiol 278: F676-F683, 2000.

31. Tomlinson DR: Mitogen-activated protein kinases as glucose transducers for diabetic complications. Diabetologia 42: 1271-1281, 1999

32. Suzuki S, Hinokio Y, Komatu K, et al: Oxidative damage to mitochondrial DNA and its relationship to diabetic complications. Diabetes Res Clin Pract 45: 161-168, 1999.

33. Kedziora K, Szram S, Komatowski T, et al: Effect of vitamin E and vitamin $C$ supplementation on antioxidative state and renal glomendar basement membrane thickness in diabetic kidney. Nephron Exp Nephrol 95: 134-143, 2003.

34. Bhatti F, Mankhey RW, Asico L, et al: Mechanisms of antioxidant and pro-oxidant effects of alpha-lipoic acid in the diabetic and nondiabetic kidney. Kidney Int 67: 1371-1380, 2005.

35. Naito Y, Uchiyama K, Aoi W, et al: Prevention of diabetic nephropathy by treatment with astaxanthm in diabetic $\mathrm{db} / \mathrm{db}$ mice. Biofactors 20: 49-59, 2004.

36. Tam FW, Riser BL, Meeran K, et al: Urinary monocyte chemoattractant protein-1 (MCP-1) and connective tissue growth factor (CCN2) as prognostic markers for progression of diabetic nephropathy. Cytokine 47: 37-42, 2009.
37. Navarro-González JF, Jarque A, Muros M, et al: Tumor necrosis factor-alpha as a therapeutic target for diabetic nephropathy. Cytokine Growth Factor Rev 20: 165-173, 2009.

38. Rivero A, Mora C, Muros M, et al: Pathogenic perspectives for the role of inflammation in diabetic nephropathy. Clin Sci (Lond) 116: 479-492, 2009.

39. Chow FY, Nikolic-Paterson DJ, Ma FY, et al: Monocyte chemoattractant protein-1-induced tissue inflammation is critical for the development of renal injury but not type 2 diabetes in obese db/db mice. Diabetologia 50: 471-480, 2007.

40. Navarro JF, Mora C, Muros M, et al: Urinary tumour necrosis factor-alpha excretion independently correlates with clinical markers of glomerular and tubulointerstitial injury in type 2 diabetic patients. Nephrol Dial Transpl 21: 3428-3434, 2006.

41. Okada S, Shikata K, Matsuda M, et al: Intercellular adhesion molecule-1-deficient mice are resistant against renal injury after induction of diabetes. Diabetes 52: 2586-2593, 2003.

42. Anatoliotakis N, Deftereos S, Bouras G, et al: Myeloperoxidase: expressing inflammation and oxidative stress in cardiovascular disease. Curr Top Med Chem 13: 115-138, 2013.

43. Crowley SD: The cooperative roles of inflammation and oxidative stress in the pathogenesis of hypertension. Antioxid Redox Signal 20: 102-120, 2014. 